\title{
Feasibility Analysis of China's Traffic Congestion Charge Legislation
}

\author{
Wang Jiyun \\ Beijing Jiaotong University Law School \\ (Beijing Jiaotong University, Beijing, China, 100044)
}

\begin{abstract}
This article is constructed based on the transportation issues which relates to introducing a congestion charge to ease chronic traffic jams. The paper first introduces the basic conception about congestion charge. Further, the supportive theory for applying congestion charge will be explored from economical and legal perspective. Lastly, summary will be presented, which analyzes and locates the advantages and deficiencies of relative legal term constitute by Chinese Civil Law.
\end{abstract}

KEYWORDS: Traffic Congestion; Legislation; Congestion Charge

\section{Introduction}

With the continuous development of urbanization and improvement of people's living standards, the motor car ownership is growing rapidly. People's demand for travel is increasing, and as a result, traffic congestion has become an "urban core problem" that most cities have generally faced nowadays. Therefore, as one of the measures to control traffic congestion, traffic congestion charge is gradually warming to become one of the most popular traffic words in recent years.

\section{Congestion Charges Legislation Overview}

A. Connotation Defining Of Traffic Congestion Charge

Currently, there are two classification methods about the "traffic congestion charge" in academia: one is in accordance with the scope of the collection, and another is in accordance with the means of implementation.

In accordance with scope of the collection, traffic congestion charge can be divided into generalized traffic congestion charges and narrow traffic congestion charges. The former refers to charging for the whole process of road use since people purchase or own vehicle; while the latter refers to an additional cost charging for the motor vehicle passing by some specific areas in specific time, to alleviate the city traffic jams pressure.

In accordance with means of implementation, traffic congestion charges can be divided into static one and dynamic one. The static traffic congestion charge refers to charging for all the vehicles that enter specific areas, without consideration of the time and spatial factors. Corresponding to static traffic congestion charge, the dynamic traffic congestion charge takes space and time into account. What's more important characteristic is with the change of 
time, the traffic congestion charge would be different, even at the same area.

\section{B. Basic Theory of Traffic Congestion Fee Legislation}

(1) Economics Theoretical Foundation

The marginal cost pricing theory in economics has always been considered as the economic theory foundation source of congestion charges. Its essence is to obtain maximum social welfare, to make marginal revenue equal to the marginal cost of transport services.

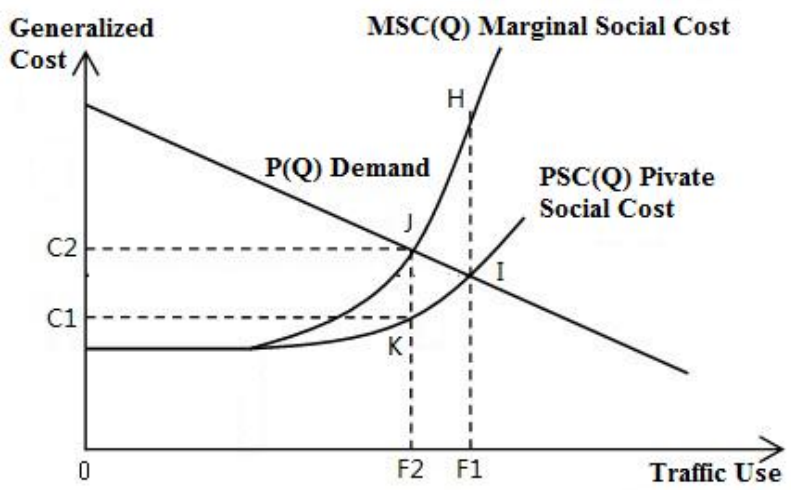

Figure 1. Road Pricing Analysis Results

Form Figure 1, we can conclude that, if everyone is charged as $\mathrm{C} 2-\mathrm{C} 1$ for road use, then balance point in the point $\mathrm{J}$, and now $\mathrm{C} 2-\mathrm{C} 1$ will be the optimal road pricing. Therefore, in order to effectively utilize the resources of road, it is necessary for government to implement road charging, until the supply and demand of traffic tends to balance.

(2) Legal Theoretical Foundation

Although traffic congestion charge is to adopt economic means to control traffic congestion, it is not only a problem of economics. Because the involved population is huge in the legislation and the implementation process, fairness, justice, efficiency, power and other factors behind it should not be ignored. Its theoretical foundation mainly originates from the theory of economics and law, and specifically manifesting as social responsibility theory.

Social responsibility theory starts from the social standard of the economic law, which advocates that satisfaction degree of the social public interests is closely link with national macro-control, market operation and social distribution behavior, and its starting point is to safeguard the public interest. Government levying traffic congestion charges is to aim at managing social issues through economic means, but social issues governance must adhere to the social responsibility theory.

Combining the two theoretical foundation of economic and law above, we believe that, the greatest value of traffic congestion charges lies in: starting from the social standard, guide the public reasonable use of public roads through economic levers, so as to make the traffic conditions achieve the optimal state as much as possible.

\section{Congestion Charges Legislation Overview}

\section{A. China's Traffic Congestion Charge Legislation Status}

At present, there are still not any nationwide applicable laws, regulations or ministerial regulations that specifically regulate traffic congestion charges levy, promulgated by national people's congress, the state council or the competent ministries. Even for the latest "Beijing 2013-2017 work program for motor vehicle emission pollution control", there is only one item in detailed list of task that mentions that traffic congestion charge will be envied in 2015 or later, but the specialized specific details on its implementation haven't been formulated yet. 


\section{B. Legal Issues of China's Traffic Congestion} Charge Legislation

(1) Lack of Sound Legal Regulations

The imperfect of laws and regulations is common problems that exist in every stage from legislation to implementation and then to enforcement, and also the problem must be solve first in traffic congestion charge levy.

Firstly, the effectiveness legislation level is low. In 2013, Beijing Municipal Government issued "“Beijing 2013-2017 work program for motor vehicle emission pollution control", which was the most explicit provisions on the levy of traffic congestion charge so far in China. However, it is not laws, administrative rules or regulations, but normative documents. In this work program, the traffic congestion charge is as a task. Because of the low-level of legislation, in the actual implementation process, it is bound to result in lack of credibility and confusion.

Secondly, the laws and regulations are imperfect. There is lack of specific laws and regulations that paly a commanding role in China's transportation law area, so the existing norms lack systematicness and continuity. For this reason, in practice, levy of traffic congestion charge can only seek applicable legal basis separately from other laws, regulations and departmental rules.

(2) Lack of Public Foundation

As a public policy, levy of traffic congestion charge is related to the vital interests of city public, thus it has the essential attribute of public policy - publicity. It is right because of the scarcity of public resources, it is inevitable that there would be contradiction in its distribution process. Therefore, the public policy of publicity nature must obtain the approval and support of the public, rather than imposing on the public.
However in our country, the existing concept of the public is that the public traffic roads are public resources, and they will not accept it if government charges them again, because they have been paying taxes to the government. On the current view of the domestic polls, most of the public is opposed to impose traffic congestion charge.

\section{Necessity of Traffic Congestion Charge in China}

Since 1990s, while China's rapid urbanization, it also means the acceleration process of urban traffic. The rapid growth of small vehicle represents the core development trend of China's urban traffic mechanization, which also brought some challenges to China's urban traffic, such as road traffic chaos, travel cost increase, etc. and this phenomenon is gradually spreading into second and third-tier cities from first-tier cities.

\section{A. Impact of Traffic Congestion on China's Social Development}

Firstly, the direct impact of traffic congestion is to increase public's travel cost and time. Increase of travel cost will not only reduce the efficiency of work, but also restrict travel people's daily activities to a certain extent.

Secondly, traffic congestion will affect the urban air quality. In recent years, the increasing of haze weather has great causal relationship with motor vehicle exhaust emissions. The vehicles in congestion traffic usually switch back and forth between stop and start, so the exhaust emissions will increase while fuel consumption increases. In addition, since the vehicles are at low speed, passage time extension will result in exhaust emission time extension. 
Finally, traffic congestion would cause a huge loss of China's economy. In 2010, the latest research results from leader Niu Wenyuan of the Chinese Academy of Sustainable Development Strategy Research Group have showed that, because of traffic congestion and management problems, 15 cities in China are losing nearly 10 billion Yuan of wealth every day.

In addition to the three obvious problems, surveys showed that traffic congestion would also lead to traffic accidents. However, once accidents happen in congested traffic, it would probably bring more congestion to the congested road. The problem of traffic congestion is becoming increasingly serious, and has become the bottleneck for the further development of the large and medium-sized city in China. Therefore, for the sake of the sustainable development of china' social economy, the task of traffic congestion governance brooks no delay.

\section{B. Significance of Traffic Congestion Fee for China's Traffic Congestion Management}

Some scholars have pointed out that, levy of traffic congestion charge in China is too early at present, and their reason is that Chinese urban traffic still cannot well undertake the group that convert from private motor vehicle into public transport. Undeniably, compared with Singapore, London or other cities, most of China's urban public transport system should be further improved, however, in recent years, public rail transport system in many large and medium-sized cities in China are developing rapidly, and Chinese government is promoting public transport priority policy all the time, which basically have the prerequisite to undertake the transferred traffic demand because of traffic congestion charge levy and to fully take the effect of traffic congestion charge levy.

It should be said that, traffic congestion charge levy is a feasible and reasonable means to manage traffic congestion phenomenon in China. Of course, the premise of this measure is the basis of perfect urban public traffic. We think, if the problems of congestion charge can be correctly handled, it will completely be able to guide the public's reasonable use of vehicle, and achieve the objective to alleviate traffic congestion.

\section{Conclusions}

Levy of traffic congestion charge involves all aspects of urban economics and society, and we should not put all the hope of traffic problem governance on traffic congestion charge levy. At the same time, we also need to consider rational road design, reasonable layout of city function, and other factors, and combine them with traffic congestion charge levy, so it can fundamentally solve the problem of urban traffic congestion in China.

We hope that, with the joint efforts of all parties, China can improve the legislation system of traffic congestion charge levy as early as possible, which will give full play to the positive role of traffic congestion fee, and improve the Chinese city traffic conditions and environmental quality, providing powerful support for enhancing development competitiveness of Chinese cities.

\section{REFERENCE:}

[1] Leape J. The London congestion charge[J]. The Journal of Economic Perspectives, 2006: 157-176.

[2] Tonne C, Beevers S, Armstrong B, et al. Air pollution and mortality benefits of the London Congestion Charge: spatial and socioeconomic 
inequalities $[\mathrm{J}]$. Occupational and Environmental Medicine, 2008, 65(9): 620-627.

[3] Ning C. The study on congestion charge in central urban district_— Take Chengdu for an example [J]. Journal of Guangzhou University (Natural Science Edition), 2009, 5: 021.

[4] Wang R. Shaping urban transport policies in China: Will copying foreign policies work?[J]. Transport Policy, 2010, 17(3): 147-152.
[5] Ye S. Research on Urban road traffic congestion charging based on sustainable development[J]. Physics Procedia, 2012, 24: 1567-1572.

[6] XU Z, OU G. Theoretical Basis and Policy Analysis on Congestion Charge[J]. China Industrial Economics, 2012, 12: 004. 